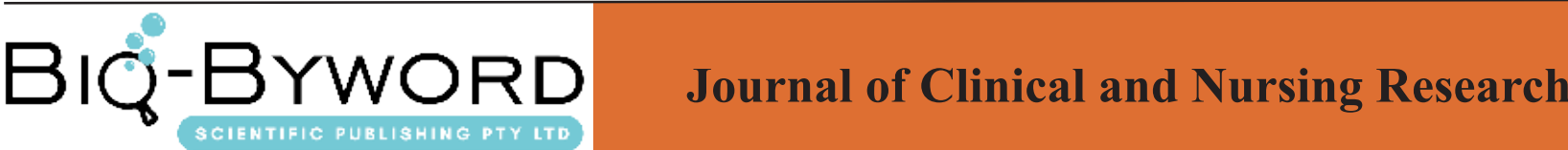

\section{Application of Non-thermal Commscope Irradiation in Preventing Com- plications of Hemodialysis Fistula}

\author{
Leng Hui \\ Jiangsu Province, Yangzhou City, armed police corps hospital, Yangzhou, Jiangsu 225000
}

\section{ARTICLE INFO}

Article history:

Published online: 15th July, 2017

Key words:

Non-thermal Commscope irradiation; prevention; hemodialysis; arteriovenous fistula

\begin{abstract}
Abstract: Objective: To ensure that the patient's treatment and control of the patient's blood in the course of long-term dialysis, in order to ensure the blood flow rate at the rate of hemodialysis in the blood vessel of hemodialysis patients, mortality rate. Methods: In many Western countries, it has been found from the experience of long-term accumulation, arteriovenous fistula (AVF) is the first choice for long-term dialysis blood patients, which has a lot of a little bit, not only from the infection rate and blood flow, which has a lot of advantages. But its existence is also a lot of complications, non-thermal Commscope irradiation is a very effective way. Results: The method was compared with the method, and it was found that $\mathrm{P}<0.05$, which was statistically significant. Conclusion: AVF is the preferred method of vascular access in patients with persistent dialysis, which is very easy to be punctured and has a very long time for maintenance. It is very important to carry out the treatment, treatment and surveillance of AVF complications and the use of means.
\end{abstract}

\section{Introduction}

In the long-term hemodialysis process, often use arteriovenous fistula as its vascular access method, this vascular pathway is to maintain the life of hemodialysis patients and the normal treatment of the channel. In the clinical work, how to extend the life of the fistula, how to ensure that patients with normal metabolism, how to fistula fibrosis and blood flow to improve and enhance, how to eliminate the needle hole and swelling and bruising and other phenomena, thus workers are worth considering about it.

\section{Materials and methods}

\subsection{General information}

There were 116 patients with arterial and venous fistula used to maintain blood games. The patients were divided into two groups. The experimental group was 58 patients. Among them, 30 were male and 28 were female. The mean age was 58 years. The control group was 58 , of which 29 cases of male patients, 
female patients in 29 cases, the average age of 59 years old. All selected 116 patients with internal fistula were mature and function well. The total amount of dialysis in the two groups was fifteen thousand times.

\subsection{Methods}

In the clinical process, the control group using the more conventional hot towel heat fistula, 24 hours after the end of dialysis, the use of hot towel hot fistula blood vessels, the temperature of the heat to the patient's tolerance as a prerequisite, the need to control patients burned or caused by fistula infection, but in the hot process, the temperature distribution faster, the need to constantly replace the towel in order to maintain a certain temperature.

The experimental group used a non-thermal Commscope irradiation method that could be used to irradiate patients with non-dialysis time or even dialysis time using the treatment instrument to expose the internal fistula and remain at least $20 \mathrm{~cm}$ with the treatment instrument during irradiation of the irradiation time of 40 minutes, one day can be one to three times the irradiation.

\subsection{Observe indicators}

1) Healing of the pinhole: the time after the puncture of the pinhole foliage to heal. 2) On the fistula and vascular embolization, fistula stenosis and occlusion, etc., the formation of embolization mainly in the appearance of induration scar, puncture process without blood, or even blood clots, fistula pulsation is very weak, need to prepare Thrombectomy; fistula blood flow caused the lack of blood vessels caused by the situation; the use of stethoscopes on the fistula for auscultation when the noise is weak or even hear, angiography can be seen in the case of vascular occlusion. 3) After dialysis, intravenous pressure, blood flow and dialysis after urea clearance index.

\section{Results}

Through the selection of the group and the relevant test, you can find in the experimental group showed some cases of subcutaneous hematoma, 6 cases, while the control group, there are 10 cases, so the experimental group of healing conditions and the effect is very good, good rate Reached $86.21 \%$, while the control group was $55.17 \%$. Therefore, according to the data show that they have differences, a statistically significant $(\mathrm{P}<0.05$ or $\mathrm{P}<0.01$ ). In addition, in the experimental group of patients after intravenous injection, found that pulse pressure was significantly decreased $(\mathrm{P}<0.01)$ and it was statistically significant. The blood flow rate was also significantly different from that of the control group ( $\mathrm{P}$ $<0.05)$.

\section{Discussion}

At present, hemodialysis patients in the course of treatment, its vascular circulation is very important, so the medical often use arteriovenous fistula to the appropriate treatment, and good fistula need to go through a long time to puncture, and repeated, so can be a substantial increase in the efficiency of its cycle, but also to provide adequate blood flow for patients, while for patients, this method is also used longer, reducing the patient's pain, through medical observation, found that But also very few complications, according to the relevant research results and experimental data show that through the use of non-thermal Kang Pu can have a better effect, if only through the hot towel can be significantly on the venous pressure and other conditions to improve, and can directly hemodialysis quality to maximize the improvement.

Non-thermal Commscope irradiation in this way the current application in China is more common, this way for the maintenance of blood vessels and the traditional way is very different, this difference is mainly manifested in deep into the deep tissue, through the laying of the way heat transfer, and this temperature does not disappear in a short time but more durable. The whole operation process more convenient, the internal fistula blood flow can be improved. The efficiency of a substantial increase in acupuncture to avoid the patients to bring bad feelings, but also the emergence of patients with vascular thrombosis and fibrosis to improve and optimize the situation, while complications such as late arterial hardening and stenosis to improve.

In summary, in patients with hemodialysis in the case of fistula in the application of non-thermal Commscope irradiation can play a very good effect to reduce the pain caused by puncture and improve hematoma healing and so have a clear understanding and application effect, so this way worthy of our country in medicine widely promoted and applied

\section{References:}

[1] Wang Haiyan, Huang Yunhui, Deng Suhong, et al. Effect and Nursing Care of Patients with Hemodialysis Fistula Treated With Hirudoid Combined with Far Infrared Therapy $[\mathrm{J}]$. Chinese Journal of Modern Nursing 201,20 (25): 3262-3264.

[2] Huang Jiarian Li Yajie, Liang Yunfang, et al. Clinical Study of Far Infrared Ray Irradiation Combined with Potato Topical Treatment for Subcutaneous He- 
matoma of Arteriovenous Fistula [J]. Journal of Nursing, 2013, 20 (12): 5-9.

[3] Li Huanbo, Ma Weiping, Jiang Xiaohong, et al. Obser- vation and Treatment of Arterial and Venous Fistula with Poor Blood Flow [J]. Practical General Practice Medicine, 2008, 6 (5): 536-537. 